\title{
Configurações
}

Revista de sociologia

\section{Território e Desenvolvimento}

\section{Ecoaldeias: práticas de cuidado a nível global e local}

Ecovillage: care practices at global and locallevel

Ecovillage: Pratiques de soins aux niveaux mondial et local

\section{Cristiana Vale Pires e Antónia Lima}

\section{(2) OpenEdition}

\section{Journals}

\section{Edição electrónica}

URL: http://journals.openedition.org/configuracoes/1935

DOI: $10.4000 /$ configuracoes. 1935

ISSN: 2182-7419

\section{Editora}

Centro de Investigação em Ciências Sociais

\section{Edição impressa}

Data de publição: 1 Junho 2013

Paginação: 99-111

ISBN: 1646-5075

ISSN: 1646-5075

\section{Refêrencia eletrónica}

Cristiana Vale Pires e Antónia Lima, «Ecoaldeias: práticas de cuidado a nível global e local »,

Configurações [Online], 11 | 2013, posto online no dia 22 setembro 2014, consultado o 24 abril 2019

URL : http://journals.openedition.org/configuracoes/1935; DOI : 10.4000/configuracoes.1935

Este documento foi criado de forma automática no dia 24 Abril 2019.

(c) CICS 


\title{
Ecoaldeias: práticas de cuidado a nível global e local
}

\author{
Ecovillage: care practices at global and locallevel \\ Ecovillage: Pratiques de soins aux niveaux mondial et local
}

Cristiana Vale Pires and Antónia Lima

\section{Introdução}

1 O movimento social das ecoaldeias tem-se expandido um pouco por todo o mundo, principalmente num contexto de crise(s) em que as pessoas procuram alternativas profissionais, de autossuficiência ou de intervenção política.

2 Este artigo procura refletir de que forma o movimento se estrutura em termos globais e locais e também as práticas de cuidado propostas num cenário de crise económica, social, política e ambiental. Baseia-se num estudo exploratório realizado entre 2010 e 2012, que se materializou na tese de Mestrado em Antropologia "Ecoaldeias: construindo alternativas" (Vale Pires, 2012), e integra o projeto "O cuidado como fator de sustentabilidade"1. Este movimento social foi analisado tendo como casos empíricos de referência o Global Ecovillage Network e as ecoaldeias Tamera e Los Angeles Ecovillage.

\section{Definindo ecoaldeias}

3 A história demonstra que o impulso para viver em comunidade é ancestral e comum a várias culturas e religiões. 0 ímpeto para viver em ecoaldeias não se define pelas formas tradicionais de pertença a grupos (por exemplo, parentesco ou etnia), mas pela partilha de valores e de uma missão, pelo que estes coletivos podem ser integrados no que Metcalf (1995) designa de comunidades intencionais.

As ecoaldeias são comunidades rurais ou urbanas de pessoas que lutam para integrar um ambiente social de apoio com um estilo de vida de baixo impacto. Para 
conseguir isto, elas integram vários aspetos de design ecológico, permacultura, construção ecológica, produção verde, energia alternativa, práticas de construção de comunidade, e muito mais. (GEN, 2012) criação de redes internacionais como o Global Ecovillage Network (GEN). Para Dawson (2010), apesar de haver uma grande diversidade no interior da família das ecoaldeias, é possível ressaltar como pilares comuns: o impulso comunitário, a iniciativa cidadã de resistência e ação, a partilha de valores, de investigação e de formação. Pelo seu carácter experimental, este projeto utiliza os avanços técnicos, científicos e informacionais de forma criativa, construtiva e sustentável, pelo que seria redutor conceptualizá-lo como um movimento back to the roots.

5 As ecoaldeias são comunidades imaginadas (Anderson, 1993), que se constroem a partir de símbolos, práticas, valores e ideias sobre o que é uma sociedade coesa, justa e sustentável. Neste sentido, mesmo que diferentes ecoaldeias proponham diferentes soluções em distintos locais, há um sentido de pertença e uma identidade partilhada interpares. humana e para a produção de novos contextos (materiais, sociais e imaginativos) fruto de contingências históricas, ambientais e imaginativas (Gupta e Fergunson, 1997). Para Kirby (2001), elas são criadas por pessoas diferentes que partilham um sentimento de descondicionamento e alienação com as sociedades convencionais e pretendem desenvolver um outro tipo de relação com os outros e com a natureza.

1998 as Nações Unidas incorporaram o conceito de ecoaldeias no Programa para o Desenvolvimento Comunitário Sustentável (SCDP), sendo este projeto posteriormente nomeado oficialmente na lista da ONU como uma das cem melhores práticas e modelos excelentes de vida para o desenvolvimento sustentável (Costa, s/a). Atualmente existem mais de quinze mil comunidades sustentáveis, sendo cerca de 1500 ecoaldeias. Algumas delas foram mesmo nomeadas como património da humanidade pela UNESCO.

\section{A emergência das ecoaldeias num contexto de globalizações}

8 As perspetivas teóricas de Antropologia da Globalização são relevantes para a análise do movimento social das ecoaldeias. Ao enfatizarem a articulação entre o global e o local e a agencialidade humana, opõem-se às perspetivas homogeneizantes, considerando, ao invés, a forma variável e diversa como os sujeitos medeiam os processos de globalização (Inda e Rosaldo, 2002). A globalização é um fenómeno multifacetado em que as dimensões económicas, sociais, políticas, ambientais, culturais e religiosas se interligam de forma complexa através de fluxos de pessoas, capitais, ideias, imagens, etc. (Appadurai, 2004). Sousa Santos (2006), por sua vez, define diferentes tipos de globalizações tendo como base o conceito de poder.

Para Negri e Hardt (2000), a globalização hegemónica remete para o sistema neoliberal e seus ideais de desenvolvimento, progresso, modernidade, consumismo. Este paradigma enfatiza o individual em detrimento do coletivo e "politiza" a economia e o consumo através da acumulação de poderes e bens e da privatização da propriedade pública (idem). Pode ainda traduzir-se em consequências de alto risco: destruição ambiental, crise económica, armas nucleares, desigualdades sociais, crescimento de super-Estados 
totalitários (Giddens, 1992), pelo que se torna necessário “(...) identificar oportunidades para a mudança dentro da sociedade - mudanças nos valores, nos estilos de vida, nas estruturas sociais - que nos irão libertar da danosa lógica social do consumismo" (idem: $65)$.

Liftin (2009) considera as ecoaldeias um movimento pós-modernista construtivo que enfatiza o organismo e os sistemas, em reação à visão atomista e mecânica do modernismo.

Combinando um ambiente social de apoio com um estilo de vida de baixo impacto, as ecoaldeias estão conscientemente a procurar criar novas formas de viver que transcendem as dicotomias da modernidade rural vs. urbano, privado vs. público, cultura vs. Natureza, local vs. global, expert vs. leigo, riqueza vs. pobreza, corpo vs. Mente. (Liftin, 2009: 127)

11 Um dos objetivos centrais das ecoaldeias é criar e experimentar formas de vida ambientalmente sustentáveis. Para Ingold (2002), o ambiente é um conceito relativo que tem existência e se desenvolve de forma contínua e construtiva com o sujeito e à sua volta. A dimensão ambiental está relacionada com dimensões políticas e económicas, pelo que, para além de questões técnicas, o discurso sobre o ambiente incorpora questões morais que moldam as narrativas e as práticas acerca do que é ser sustentável. "O ambiente está a tornar-se a questão política da nossa era, em que têm vindo a emergir novas formas de moralidade, sociabilidade e governança" (Leach e Fairhead, 2012: 275). Este discurso moralista tem implícitas narrativas sobre colapso ecológico e salvação pela redenção (mudança de relação com o meio ambiente), pelo que muitas vezes o ambientalismo se configura não apenas como uma causa mas também como uma religião (Garreau, 2010).

\section{Ecoaldeias - do global ao local}

\section{GEN - A comunidade de comunidades}

12 O estudo exploratório realizado com o GEN baseou-se na observação participativa, durante a sua conferência "Strategies for Resilience", realizada na ecoaldeia Tamera em 2011, numa entrevista semiestruturada à presidente do movimento e na observação online através de uma mailing list, em que residentes de várias ecoaldeias discutem temas $\mathrm{e}$ preocupações comuns.

O GEN é uma plataforma transnacional de ecoaldeias que visa "apoiar a criação experimental e preservação de estilos de vida de baixo impacto a nível global" e "promover o diálogo e a troca, construir solidariedade e parcerias em todas as fronteiras", para "a emergência de uma fonte de sabedoria diversa mas globalmente compartilhada para uma vida sustentável” (GEN, 2012). De acordo com Macaco Tamerice 3 , "o GEN cria o sentimento de se fazer parte do mesmo movimento e oferece a possibilidade de partilhar práticas, sabedorias, conhecimentos sobre permacultura, energia renovável, mas também a consciência de que juntos podemos fazer mais".

Para além de agir como uma base de dados, o GEN promove vários workshops, conferências de partilha de experiências e conhecimentos e iniciativas de advocacy com decisores políticos que visam validar as ecoaldeias enquanto alternativa viável. A este respeito, Macaco Tamerice considera que "as ecoaldeias estão muito mais vocacionadas para ser, sentir, viver do que para tentar influenciar o mundo exterior. Este é o nosso maior 
desafio, como é que podemos mudar a nossa imagem para comunicar com o mundo exterior sem perder a nossa identidade".

Como se poderá perceber pela descrição de dois exemplos locais, apesar da filosofia e missão partilhadas, cada ecoaldeia tem a sua própria idiossincrasia, atendendo à visão dos seus fundadores e aos contextos em que emergem.

\section{Tamera - Biótipo de Cura}

16 Tamera é uma ecoaldeia situada no Alentejo e que surge com o objetivo de criar "um modelo para o futuro" (Dregger, 2010).

Considerando as atuais condições do mundo, não é possível ver um perspetiva convincente para a convivência não violenta no nosso planeta. Para criar condições favoráveis, terão de surgir centros nos quais será possível pensar e desenvolver exemplos práticos sobre a convivência não violenta do ser humano com todas as cocriaturas. Tamera existe para apoiar o surgimento desses centros (Dieter Duhm, in Dregger, 2010: 10)

Tamera foi fundada em 1995 no sudoeste de Portugal e ocupa 134ha num terreno árido no distrito de Beja. Tem como objetivo ser um modelo de futuro ecologicamente sustentável, autossuficiente e descentralizado a nível regional e social. Neste sentido, investiga temas relacionados com o ambiente, a organização social não hierarquizada e o relacionamento interpessoal e afetivo. Dependendo da fase do ano, residem em Tamera cerca duzentas pessoas, entre as quais residentes, estudantes e visitantes. Há uma grande variabilidade cultural em Tamera: 35 nacionalidades, sendo prevalente a nacionalidade alemã.

A etnografia realizada em Tamera decorreu em três períodos de observação participante: um dia aberto a visitantes em 2010, estadia de uma semana durante a conferência do GEN em 2012 e estadia durante uma semana em março de 2012.

o trabalho político em Tamera está fortemente relacionado com a espiritualidade, promovendo peregrinações e meditações para a criação de um "campo morfogenético pela paz"4 . Barbara Kovats ${ }^{5}$ referiu que havia o plano de construir vários Biótipos de Cura (Tamera é o Biótipo I), que funcionariam como agulhas de acupunctura para curar a Terra. Por esse motivo, Tamera presta apoio a situações de crise, como é o caso da comunidade San José de Apartado na Colômbia, fortemente pressionada pelo narcotráfico, e promove a criação de centros de paz em Israel/Palestina.

Para legalizar os sistemas estruturais e financeiros, foi necessário criar o ILOS - Peace Research Ltd., uma cooperativa gerida por todos os residentes, proprietária de Tamera e que reinveste os seus lucros nos projetos comunitários. O ILOS divide-se em dois subgrupos: a Associação para um Mundo Humanitário e a Associação G.R.A.C.E (Grupo para a Reconciliação em Áreas de Crise e Educação). A primeira associação inclui as áreas de organização, logística e investigação, e a G.R.A.C.E é responsável pela educação e cursos.

21 Em termos económicos, Tamera não é autónoma. O dinheiro é necessário para a concretização dos projetos e atividades, e deriva das rendas, cursos e donativos. Neste sentido, para residir em Tamera, é preciso investimento em termos de tempo e dinheiro, pois implica participar em cursos de formação, trabalho voluntário, visitas e estadias mais ou menos prolongadas. Portanto, os residentes ou são de classe média-alta ou têm trabalhos sazonais fora da ecoaldeia para suportar a renda (10€/dia), sendo a maior parte oriundos do Norte da Europa. 
Em Tamera, há interações com as populações locais.

Tamera está a desenvolver um modelo para uma autonomia regional de géneros alimentares em cooperação com vizinhos, proprietários de cultivo biológico e pequenos produtores do Alentejo, criando uma rede de distribuição de produtos alimentares regionais (...). Desta forma as comunidades e aldeias podem tornar-se economicamente independentes. (Dregger, 2010: 41)

Em termos práticos, esta ecoaldeia é constituída por microcomunidades, cada uma a trabalhar determinados temas/projetos. Assim há a Aldeia Solar (tecnologia e ecologia), Aldeia da Luz (arte, medicina tradicional, a mulher), Espaço das Crianças (educação alternativa e crianças em comunidade), Campus Global (zona de estudantes e visitantes), Ashram Político e Instituto para a Paz Global (política e ativismo). Cada zona tem as suas próprias estruturas comunitárias físicas (por exemplo, cozinhas e casa de banho) e sociais (fóruns, reuniões). Os residentes distribuem-se pelas várias zonas em função das suas competências e preferências.

Os projetos de ecologia trabalham temas como a água, o solo, a reflorestação, a reserva de sementes, a criação de paisagens comestíveis, a vivência em paz entre o homem e a natureza. A água é um tema central em Tamera, sendo venerada a nível espiritual e incorporada nos projetos políticos. A paisagem aquática de Tamera é constituída por um conjunto de lagos (reservatórios de água da chuva), construídos seguindo os princípios de permacultura, que permitem suprir as necessidades de água e criar condições para a "cura" da terra. No Campo Experimental da Aldeia Solar são testadas instalações solares descentralizadas que produzem energia térmica, elétrica e mecânica. Outra área de interesse é a bioconstrução através de materiais naturais e técnicas tradicionais do Alentejo.

O amor, a sexualidade e a relação são considerados questões políticas, visto que o ciúme, a competição e o medo de perda se aplicam também à relação com a Natureza. Neste sentido, enfatiza-se o "amor livre": amor sem ciúmes, sexualidade sem medos, verdade e fidelidade que se mantém mesmo se houver amor e desejo por outros. Esta política de desapego aplica-se também à educação das crianças. O Espaço das Crianças é constituído por vinte crianças, dos 0-14 anos (residentes em Tamera ou na vizinhança), e representa um espaço de segurança e confiança protegido das expectativas dos adultos.

A espiritualidade assume um papel fundamental, funcionando como o "cimento" que "sacraliza" todo o projeto. 0 "coração de Tamera" é representado por um círculo de pedras idealizado por Sabine Lichtenfels e inspirado no Cromeleque dos Almendres.

Os processos de decisão, ao nível das equipas e áreas, baseiam-se em democracia participativa; ao nível da ecoaldeia as decisões são tomadas pelo Conselho da Comunidade - estrutura de decisão representativa, constituída por líderes e coordenadores de equipa. Para estimular o fortalecimento das relações em comunidade e a transparência na comunicação, usam o Fórum, uma ferramenta social que se baseia na comunicação intergrupal e na escuta ativa de cada participante.

\section{Los Angeles Ecovillage (LAEV) - o modelo urbano}

Apesar de a grande maioria das ecoaldeias se construir em ambiente rural, aparecem cada vez mais exemplos da sua aplicação em meio urbano. "O objetivo de uma ecoaldeia urbana é verificar o que pode ser feito localmente para melhorar os locais em que nós já vivemos, de forma a nutrirem o nosso bem-estar social, mental e físico enquanto nos fornecem os 
recursos para satisfazer as nossas necessidades diárias" (Sizemore, 2004: 16). As ecoaldeias urbanas podem ser um conjunto de casas ou edifícios, casas partilhadas ou um bairro inteiro.

A LAEV está localizada em Los Angeles, uma megacidade caracterizada não apenas pela densidade populacional e suas problemáticas (por exemplo, desigualdades sociais, criminalidade) mas também pela diversidade cultural e pela sua ligação a estilos de vida alternativos e ativismo.

A etnografia realizada em LAEV baseou-se em observação participante durante um mês de residência na ecoaldeia e entrevistas semiestruturadas a alguns residentes.

31 A LAEV está localizada em Bimini and White House Place neighborhood, um bairro multicultural de classe média-baixa, com cerca de quinhentos habitantes. Foi no interior deste bairro que um grupo de quarenta pessoas se organizou intencionalmente para formar a LAEV e experimentar um estilo de vida que visa "resolver estas questões complexas demonstrando uma forma diferente de estar na nossa cidade" (LAEV, 2012). Esta comunidade foi fundada pelo CRSP (Cooperative Resources and Services Project), organização educativa e de desenvolvimento comunitário sem fins lucrativos, criada pelos fundadores da LAEV em 1980, que serve de centro de recursos para pequenos bairros e grupos que visam formar comunidades intencionais ou tornar-se sustentáveis.

De acordo com Lois Arkin, 74 anos, fundadora da LAEV, quando o CRSP iniciou a criação da ecoaldeia, os residentes mal se conheciam, temiam-se entre si e não havia atividades comuns no bairro. Assim,

Os residentes na ecoaldeia pretendem adquirir e demonstrar estilos de vida eficazes e de baixo impacto, para reduzir a necessidade do governo, e para aumentar a autoconfiança do bairro em áreas como subsistência, produção de comida, uso de energia e água, habitação a preço acessível, trânsito, atividades recreativas, redução dos desperdícios e educação. (LAEV, 2012)

O CRSP comprou o edifício com quarenta apartamentos (117 Bimini Place) em 1996 e o edifício adjacente com oito apartamentos em 1999 através de um empréstimo cedido pelo seu fundo ecológico de empréstimo rotativo (ELF - Ecological Revolving Loan Fund). Este fundo é conseguido graças a financiadores privados e à renda paga pelos moradores, o que permite manter as propriedades e as rendas baixas. o dinheiro do ELF é gerido coletivamente, mas regra geral destina-se a investimentos como: a eco-reabilitação dos apartamentos do CRSP; a aquisição de novas propriedades para a ecoaldeia e para fornecer capital "semente" para o desenvolvimento de novos negócios verdes dentro da ecoaldeia (LAEV, 2012).

Os residentes da ecoaldeia estão a demonstrar padrões de vida de baixo impacto enquanto aumentam a qualidade de vida do bairro. Eles trabalham para efetivamente integrar os aspetos sociais, económicos e ecológicos da vida em comunidade. (LAEV, 2012)

Para cumprir este objetivo:

Temos uma reunião geral todas as semanas, às vezes temos pessoas que vêm cá falar, como ontem o workshop de chicken tractor... Temos retiros, momentos em que alguém cozinha para todos fazemos compostagem, promovemos a cultura da bicicleta, temos uma cooperativa de produtos biológicos disponível também para quem não vive na comunidade, reciclamos água, a água cinzenta claro. Algumas pessoas são responsáveis pelo banco de tempo, outras estão a participar e a cozinhar para o Occupy LA, o Erik participa na Passenger Union para promover melhores transportes em LA, um outro membro foi o fundador da Ciclovia que é 
uma via com cinquenta milhas onde podes andar de bicicleta, eu organizo o festival

Ecomaia que promove o ambiente e a cultura maia... (Julio Santizo ${ }^{6}$ )

\section{participar nas atividades da ecoaldeia durante dezoito meses e aceitar os objetivos e} regras.

\section{0 cuidado como fator de sustentabilidade}

37 Na pós-modernidade a motivação para cuidar aplica-se também a causas globais, definindo-se "como exercício fáctico da responsabilidade - (...) que se desenvolve em ações concretas, a diferentes níveis institucionais e políticos, dando novos contornos àquilo que constitui a 'democracia', não tanto enquanto regime político, mas quanto a título de imperativo ético" (Borges Duarte, 2011: 44).

As ecoaldeias enfatizam princípios de ética ambiental, complementando o valor instrumental da Natureza com o seu valor intrínseco. "O primeiro refere-se ao valor das coisas enquanto meios para alcançar fins, enquanto o segundo diz respeito ao valor das coisas enquanto fins elas próprias independentemente se são também meios úteis para outros fins" (Brennan e Yeuk-Sze, 2011).

As ecoaldeias tentam retomar um contacto e uma troca harmoniosa com a natureza. Fazemos todos parte de Gaia, este planeta. É importante retomar uma relação sem exploração e sem o magoar. Para nós isso signifi ca usar energia renovável, diminuir a nossa pegada em $\mathrm{CO} 2$, cultivar de forma orgânica a nossa própria comida, permacultura. (Macaco Tamerice)

39 A lógica presente nas ecoaldeias reflete uma modernidade técnica e acima de tudo ética que não se reduz à realidade humana mas se abre ao campo mais amplo da Natureza. Cuidar da Natureza é, em última instância, cuidar do mundo e da espécie humana. "A natureza é forte e irá resistir, a pergunta é se os humanos conseguirão sobreviver a todas as mudanças que irão ocorrer. Eu sou positiva e acredito que a humanidade vai encontrar soluções" (Macaco Tamerice). Assenta, portanto, na compreensão e na reflexão a respeito do mundo em que vivemos, mas também no compromisso e na responsabilidade de agir para preservar o ser humano e o mundo natural (Bartholo, in Santos Júnior, 2006). Desta forma a sustentabilidade é um conceito que se centra nas pessoas para a conservação da natureza, visto que terá de se melhorar a qualidade de vida humana enquanto se mantêm a vitalidade e a diversidade dos ecossistemas (Jackson, 2009).

A vivência em ecoaldeias facilita este processo, principalmente pelo reforço no comprometimento e pela natureza comunitária e design, promotores de estilos de vida mais verdes e sustentáveis.

Nas ecoaldeias estamos comprometidos em maximizar a nossa pegada positiva e minimizar a pegada negativa nos sistemas naturais. Para isso nós fazemos compostagem para minimizar os nossos desperdícios, devolvendo restos alimentares ao solo e tentamos minimizar o consumo. (Yuki, 2011)

41 Estas práticas inspiram-se no design em permacultura. Este conceito foi formalmente definido em 1978, na Austrália, por Bill Mollison e David Holmgren. "Permacultura 
(cultura permanente) diz respeito a um design consciente e à manutenção de ecossistemas agrícolas produtivos que incluem a diversidade, estabilidade e resiliência dos ecossistemas naturais" (Mollison, 1990: xi). Para isso, interliga clima, plantas, animais, ciclos de nutrientes, solo, gestão da água e necessidades humanas em unidades produtivas e eficientes, para trabalhar em cooperação com a Natureza. Relaciona-se com valores e ética voltados para o ambiente e enfatiza o sentido de responsabilidade de cuidar, reabilitar e criar. As bases éticas da permacultura são: cuidar da Terra, cuidar das pessoas, colocar limites à população e ao consumo. É uma filosofia holística que propõe formas alternativas de gestão social, económica e ambiental, produção de energia, educação e saúde.

Aliada ao design em permacultura, as ecoaldeias adotam a "simplicidade voluntária" como fi losofi a de vida para viver de forma mais sustentável (The Simplicity Forum, 2011). Neste contexto, o conceito de simplicidade não é fácil de definir, pois pode ter múltiplas motivações, formas e expressões (por exemplo, consumir menos, reutilizar). A grande maioria das ecoaldeias aplica práticas como o slow food ${ }^{7}$, Boicote e Buycott que se referem à procura consciente e intencional de produtos e/ou serviços percebidos como social e ambientalmente sustentáveis (Wilson, 2010).

Consumir menos aumenta o bem-estar subjetivo, e as pessoas parecem ser mais felizes (Jackson, 2009), o que contraria as perspetivas que defendem que um comportamento ambientalmente responsável implica autossacrifício. Para Brown e Kasser (2005), o bemestar pessoal é complementar com o bem-estar planetário, e relaciona-se com um "cultivo" maior dos valores intrínsecos (crescimento pessoal, envolvimento na comunidade) e menor dos valores extrínsecos (sucesso financeiro, popularidade). Neste sentido, experienciam o que Hinton e Goldman (2010) designam por hedonismo alternativo, isto é, fontes satisfatórias e sustentáveis de identidade, criatividade e significado que se encontram fora do mercado convencional. Cuidar de uma horta, andar de bicicleta, reutilizar materiais, para além de satisfazerem algumas necessidades, são atividades de lazer. "Para mim não é só fazer sem, nem a ideia de que temos de sofrer. Andar de bicicleta é divertido e faz-me ver a cidade de uma outra forma, vês pessoas, a vizinhança e ainda por cima é bom para a saúde, para o ambiente, para tudo" (Yuki).

Atualmente, dada a redução do papel do Estado social, o cuidado a partir dos laços comunitários e redes informais pode assumir um importante papel.

É triste quando o governo apoia o exército contra outros países, quando discrimina, quando corta toda a segurança social que o nosso dinheiro pagou antes.

Por isso precisamos de nos organizar em comunidades para ter saúde. (...) Em 2005 tive cancro no cólon e senti como se o céu me caísse na cabeça, mas foi bom porque a comunidade me ajudou com comida, entretenimento, apoio, acompanhando-me ao médico. (Júlio Santizo)

\section{Conclusão}

O movimento social das ecoaldeias é constituído por uma grande variabilidade de práticas e projetos, cuja idiossincrasia depende de fatores económicos, sociais, culturais, políticos e ambientais locais. É também composto por espaços transnacionais ou nodais, onde coabitam pessoas de múltiplas nacionalidades, que aliam a procura de um estilo de vida mais simples e comunitário à vontade de contribuir para uma sociedade mais justa, inclusiva e ambientalmente sustentável. Neste sentido são contextos onde os níveis local e 
global e as motivações individuais e coletivas estão em constante articulação e se potenciam mutuamente.

Através dos exemplos analisados, procuramos argumentar que nas ecoaldeias se desenvolvem e experimentam práticas de cuidado para com o ser humano e o meio ambiente. As ecoaldeias assumem-se como laboratórios ou modelos que experimentam possibilidades de superação das crises económicas, sociais e ambientais contemporâneas. Apesar deste seu potencial, e de em algumas áreas, efetivamente, fugirem às lógicas do modelo hegemónico (por exemplo, o consumo), a maior parte das ecoaldeias necessita de fi nanciamento. Este facto coloca em causa o seu princípio de autossuficiência, visto que para se construírem estão dependentes de dinheiro, tornando-as espaços eco-chic (ESF, 2011), onde apenas poderá residir, visitar e aprender quem tem disponibilidade económica (Vale Pires, 2012). Por outro lado, e usufruindo das potencialidades das globalizações, as ecoaldeias difundem valores morais sobre o que é um estilo de vida e uma organização social adequada para fazer frente aos desafios da humanidade. Considerando a reflexão de Howell (2003) a respeito da ajuda humanitária a crianças, as ecoaldeias apresentam uma agenda normativa. Agem como missionários que difundem ideias e valores ocidentais sobre o que é ambientalismo, justiça social e paz, sem questionar a "retidão" das suas propostas, apesar de procurarem considerar "a multiplicidade de soluções humanas para questões existenciais cosmologicamente construídas" (idem: 213).

Em jeito de conclusão, e considerando que as ecoaldeias podem não ser "modelos para o futuro" por não representarem a solução possível, desejada ou considerada pela maior parte das pessoas, estruturam-se como "incubadoras" com potencial para ativar um efeito de bola de neve de inspiração e influência para a aquisição e vivência de estilos de vida mais sustentáveis.

\section{BIBLIOGRAPHY}

ANDERSON, Benedict (2005), Comunidades imaginadas: reflexões sobre a origem e a expansão do nacionalismo, Lisboa, Edições 70.

APPADURAI, Arjun (2004), Dimensões culturais da globalização, Lisboa, Teorema.

BORGES-DUARTE, Irene (2011), "Responsabilidade: Fundamento Básico da Democracia”, in Fundação Cuidar o Futuro (org.), Cuidar a democracia, cuidar o futuro, Lisboa, FCT: 37-44.

BRENNAN, Andrew e YEUK-SZE, Lo (2011), “Environmental Ethics”, in Edward N. Zalta (ed.), The Stanford Encyclopedia of Philosophy, Metaphysics Research Lab, CSLI, Stanford University [online]. Disponível em: http://plato.stanford.edu/entries/ethics-environmental/ [Consultado em: 22/09/2011].

BROWN, Kirk Warren e KASSER, Tim (2005), “Are Psychological and Ecological Well-being compatible? The role of values, mindfulness, and lifestyle", Social Indicators Research, 74: 349-368 [online]. Disponível em: http://www.thesimplicityforum.org/ [Consultado em: 13/08/2011]. 
COSTA, Ricardo André (s/a), "Ecoaldeia, um conceito necessário no panorama das alterações climáticas”, Departamento de Ambiente e Ordenamento, Universidade de Aveiro [online]. Disponível em: http://www.apea.pt/xFiles/scContentDeployer_pt/docs/Doc159.pdf [Consultado em 24/02/2011].

DAWSON, Jonathan (2010), Ecoaldeias: Novas fronteiras para a sustentabilidade, Edições Sempre-EmPé, Ed. 24.

DREGGER, Leila (2010), Tamera: Um modelo para o futuro, Verlag Meiga, Belzig.

EUROPEAN SCIENCE FOUNDATION - ESF (2011), “Call for Papers Conference: Eco-chic Connecting ethical, sustainable and elite consumption" [online]. Disponível em: http://www.esaconsumption. org/about.php. [Consultado em 11/12/2011].

GARREAU, Joel (2010), “Environmentalism as Religion”, The New Atlantis: A Journal of Technology and Society: 61-74.

GIDDENS, Anthony (2002), As Consequências da Modernidade, (2. ${ }^{\mathrm{a}}$ Ed.), Oeiras, Celta Editora.

GLOBAL Ecovillage Network [online]. Disponível em: http://gen.ecovillage.org/, [Consultado em 18/03/2012].

GUPTA, Akhil e FERGUNSON, James (1997) "Culture, Power, Place: Ethnography at the End of an Era", in Akhil Gupta e James Fergunson (orgs.) Culture, Power, Place: Explorations in Critical Anthropology. Durham, Duke University Press: 1-32.

HINTON, Emma D. e GOODMAN, Michael K. (2010) “Sustainable consumption: developments, considerations and new directions", in Michael R. Redcliff e Graham Woodgate (org.), The International Handbook of Environmental Sociology, 2. ${ }^{a} \mathrm{Ed}$, Cheltenham, Edward Elgar Publishing Limited: 245-261.

HOWELL, Signe (2003), “The Diffusion of Moral Values in a Global Perspective”, in Thomas Hylland Eriksen (ed.), Globalisation: Studies in Anthropology. Londres: Pluto Press.

INDA, Jonathan Xavier e ROSALDO, Renato (2001), “Introduction: A World in Motion", Jonathan Xavier Inda e Renato Rosaldo (orgs.) The Anthropology of Globalization: A Reader, Malden: Blackwell Publishers: 1-34.

INGOLD, Tim (2002), The Perception of the Environment: Essays on Livelihood, Dwelling and Skill, Londres: Routledge, 13-26: 61-77.

JACKSON, Tim (2009), Prosperity without growth? The transition to a sustainable economy, Sustainable Development Commission [online]. Disponível em: http://www.sd-commission.org.uk/ publications.php?id=914 [Consultado em: 28/01/2012].

KIRBY, Andy (2003), "Redefining social and environmental relations at the ecovillage at Ithaca: a case study", Journal of Environmental Psychology, 23: 323-332.

LEACH, Melissa e FAIRHEAD, James (2012), “Environment and Society: Political Ecologies and Moral Futures", Richard Fardon et al. (orgs.), The Sage Handbook of Social Anthropology, Vol. 2, Thousand Oaks: Sage Publications: 275-285.

LIFTIN, Karen (2009), "Reinventing the future: The global ecovillage movement as a holistic knowledge community”, in Gabriela Kütting e Ronnie Lipschutz (orgs.), Environmental Governance: Power and Knowledge in a local-global world, Routledge: 124-142.

LOS ANGELES ECOVILLAGE [Online]. Disponível em: http://www.laecovillage.org/ [Consultado em $21 / 01 / 2012]$ 
METCALF, William James (1995), From Utopian Dreaming to Communal Reality: cooperative lifestyles in Australia, Sydney, University of New South Wales Press.

MOLLISON, Bill (1990), Permaculture - A practical guide for a sustainable future, Washington, DC: Island Press, 1-69 e 505-558.

NEGRI, Antonio e HARDT, Michael (2000), Empire, London, Harvard University Press.

SIZEMORE, Steve (2004), Urban Eco-villages as an Alternative Model to Revitalizing Urban Neighborhoods: The Eco-village Approach of the Seminary Square/ Price Hill Eco-village of Cincinnati, Ohio, Dissertação de Mestrado em Community Planning, College of Design, Architecture, Art, and Planning, Division of Research and Advanced Studies of the University of Cincinnati [online]. Disponível em: http://etd.ohiolink.edu/view.cgi?acc_num=ucin1085610666 [Consultado em 09/03/2012].

SANTOS JÚNIOR, Severiano José (2006), “Ecovilas e Comunidades Intencionais”, comunicação apresentada em III Encontro das ANPPAS (Associação Nacional de Pesquisa e Pós-Graduação em Ambiente e Sociedade), 23 a 26 de maio de 2006, Brasília [online]. Disponível em: http://tinyurl.com/12sqs56 [Consultado em: 2/04/2011].

SOUSA SANTOS, Boaventura (2006), "Globalizations", Theory, Culture \& Society, 23: 2-3 e 393-399.

TAMERA [online]. Disponível em: http://www.tamera.org/index.html [Consultado em 15/05/2012].

THE Simplicity Forum [online]. Disponível em: http://www.thesimplicityforum.org/ [Consultado em: 13/08/2011].

WILSON, Bradley R. (2010), Between Market and Movement: The fair trade coffee 'buycott' in the United States and Nicaragua, Dissertação de Doutoramento, Graduate School-New Brunswick Rutgers, The State University of New Jersey [online]. Disponível em: http://mss3.libraries.rutgers.edu/dlr/ showfed.php?pid=rutgers-lib:26582 [Consultado em 13/02/2012].

VALE PIRES, Cristiana (2012), Ecoaldeias: Construindo Alternativas - Estudo exploratório do movimento social das Ecoaldeias através do Global Ecovillage Network, Tamera e Los Angeles Ecovillage, Dissertação de Mestrado em Antropologia: Globalização, Migrações e Multiculturalismo, Instituto Superior das Ciências e do Trabalho, Universidade de Lisboa.

\section{NOTES}

\section{FCT PTDC/CS-ANT/117259/2010 IR Antónia Lima}

2. Associação dinamarquesa fundada em 1987 para apoiar a transição para um futuro mais sustentável através de subsídios e iniciativas proativas. Mais informações em: www.gaia.org

3. Presidente do GEN e residente na ecoaldeia Damanhur, entrevista realizada durante a conferência do GEN em Tamera.

4. Sistema holístico de informação e comunicação para despertar a memória coletiva das sociedades humanas, promovendo a substituição de hábitos antigos (guerra, competição) por outros mais adaptativos (paz, cooperação).

5. Residente em Tamera desde o início do projeto, Coordenadora da Aldeia Solar.

6. Guatemaleco, residente em LAEV há onze anos.

7. Movimento que articula produtores e consumidores em cadeias de proximidade que apoiem dietas mais saudáveis, socialmente justas e ambientalmente sustentáveis. 


\section{ABSTRACTS}

Os processos de globalização, a evolução dos sistemas comunicacionais e informacionais e as atuais crises (ambiental, económica e política) têm motivado algumas pessoas a refletir e construir diferentes organizações sociais e estilos de vida. Neste artigo é apresentado o movimento social das ecoaldeias, explorando as suas dimensões globais, através do Global Ecovillage Network, e locais, através das práticas de Tamera e Los Angeles Ecovillage. A partir da descrição de ambas as dimensões deste movimento social, argumenta-se que o cuidado é transversal ao design e experimentação na construção de modelos de vida alternativos para si e para o mundo, sendo portanto um fator de sustentabilidade.

The globalization processes, the evolution of the communicational and informational systems and the current crisis (environmental, economic and politic) are motivating some people to reflect and build different social organizations and life styles. With this article we aim to introduce the ecovillages social movement, exploring their global dimensions through Global Ecovillage Network and their local practices through Tamera and Los Angeles Ecovillage. With this background we argue that, in this social movement, care is transverse to the design and experimentation of alternative lifestyles to themselves and to the other, so it can be considered a sustainability factor.

Les processus de mondialisation, le développement des systèmes communicationnels et informationnels et les crises actuelles (environnementales, économiques et politiques) ont motivé certaines personnes de réfléchir et de construire des organisations sociales et des styles de vie. Dans cet article nous vous présentons le mouvement social des écovillages, à travers de l'exploration de leurs dimensions mondiales par l'intermédiaire du Global Ecovillage Network, et locales à travers les pratiques de Tamera et Los Angeles Ecovillage. Grâce à la description de ces deux dimensions de ce mouvement nous soutenons que que l'aide sociale est transversale à la conception et à l'expérimentation dans la construction de modèles alternatifs de vie pour euxmêmes et pour le monde, étant donc un facteur de durabilité.

\section{INDEX}

Mots-clés: mondialisations, mouvements sociaux, éthique environnementale, soins

Keywords: Globalizations, social movements, environmental ethics, care

Palavras-chave: Globalizações, movimentos sociais, ambientalismo, cuidado

\section{AUTHORS}

\section{CRISTIANA VALE PIRES}

Doutoranda de Antropologia no ISCTE-IUL - Instituto Universitário de Lisboa e investigadora no CRIA - Centro em Rede de Investigação em Antropologia

cris.vpires@gmail.com 


\section{ANTÓNIA LIMA}

Professora auxiliar no Departamento de Antropologia no ISCTE-IUL e investigadora no CRIA Centro em Rede de Investigação em Antropologia

antonia.lima@iscte.pt 